\title{
Adsorbent-SERS technique for determination of plant VOCs from live cotton plants and dried teas
}

\author{
Jinhyuk Park 1,*, J. Alex Thomasson ${ }^{1}$, Cody C. Gale ${ }^{2}$, Gregory A. Sword ${ }^{2}$, Kyung-Min
} Lee ${ }^{3}$, Timothy J. Herrman ${ }^{3}$, Charles P.-C. Suh ${ }^{4}$

1. Department of Biological and Agricultural Engineering, Texas A\&M University, College Station, TX 77843, USA

2. Department of Entomology, Texas A\&M University, College Station, TX 77843-2475, USA

3. Office of the Texas State Chemist, Texas A\&M AgriLife Research, Texas A\&M University System, College Station, Texas 77841, USA

4. Insect Control and Cotton Disease Research Unit, USDA, ARS, 2771 F\&B Road, College Station, TX 77845

* Corresponding author

E-mail address: jijinbba202@tamu.edu (Jinhyuk Park)

Tel: +1-979-224-7055

\section{Supplementary}

\section{S1 Gas concentration approximation}

1. Maximum limit based on the assumption that the vapor was generated due to its own vapor pressure

$$
\therefore \quad C_{V O C, \max }[p p m v]=\frac{V_{V O C}}{V_{\text {Total }}} \times 10^{6}=\frac{P_{V O C}}{P_{\text {atm }}} \times 10^{6}
$$

$$
V_{V O C}=\text { volume of VOC }[L], \quad V_{\text {Total }}=\text { total volume of the chamber }=120 \times 10^{-3}[L]
$$

$$
P_{\text {atm }}=\text { standard atmosphere }=1[\mathrm{~atm}], \quad P_{V O C}=\text { vapor pressure of VOC }[\mathrm{atm}] \text {, }
$$

$$
\mathrm{R}=\text { Ideal gas constant }=0.0821\left[\frac{\mathrm{L} a t m}{K \text { mole }}\right], \quad \mathrm{T}=\text { Absolute temperature }
$$

$$
=298[\mathrm{~K}] \text { at } 25{ }^{\circ} \mathrm{C}
$$

\begin{tabular}{ccc}
\hline VOC & Vapor pressure (atm) & $C_{V O C, \min }[p p m v]$ \\
\hline Linalool & 0.00022 & 220 \\
Cis-3-hexen-1-ol & 0.0012 & 1200 \\
Methyl salicylate & 0.000045 & 45 \\
\hline
\end{tabular}

Table S 1. Concentration approximation for three VOCs 
(a)<smiles>Cc1ccccc1</smiles>

A: Any element except $\mathrm{H}$ (b)

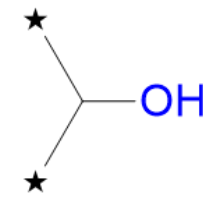

Secondary<smiles>C[C](C)O</smiles>

Tertiary $\star$ : Any attachments

Figure S 1. Possible molecular groups (a) Aromatic component with ortho-disubstituted (b) any secondary or tertiary alcohol

\section{S3 Raman spectra for standards}

All Raman spectra for standards except for ocimene were generated from commercial libraries (KnowItAll Informatics System 2018), and the spectra for ocimene was generated from its standard.

(a)
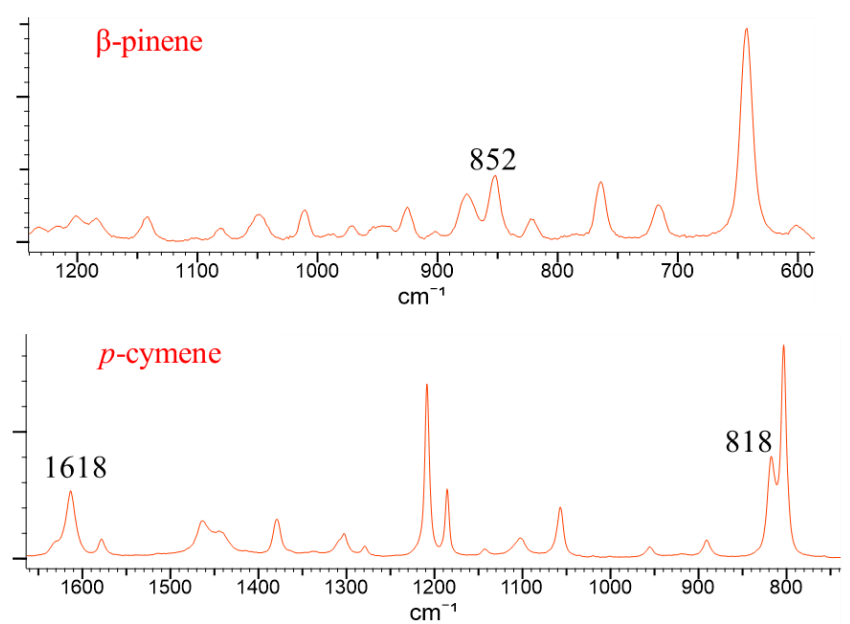

(b)
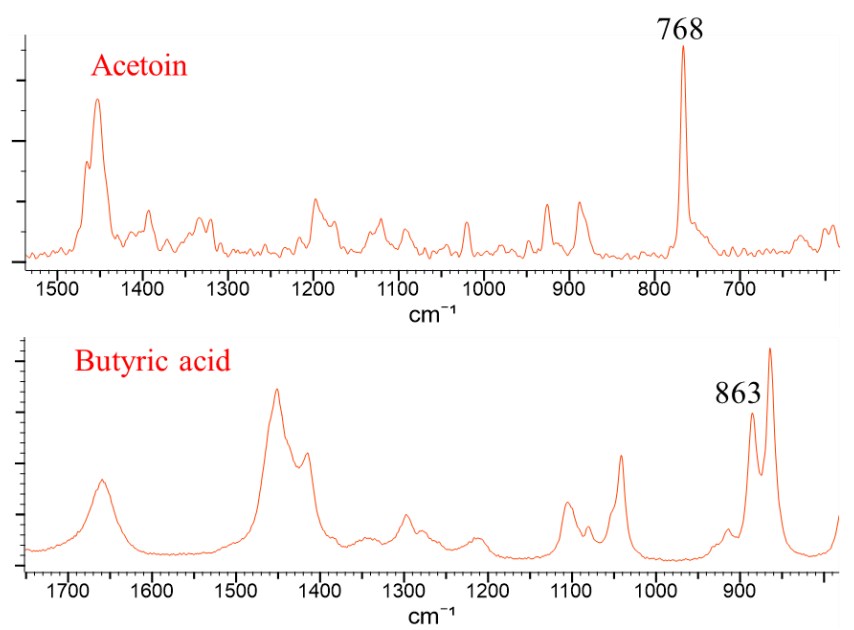

Figure S 2. Raman spectra for standards relevant to (a) Earl grey VOC, and (b) Rooibos VOC 

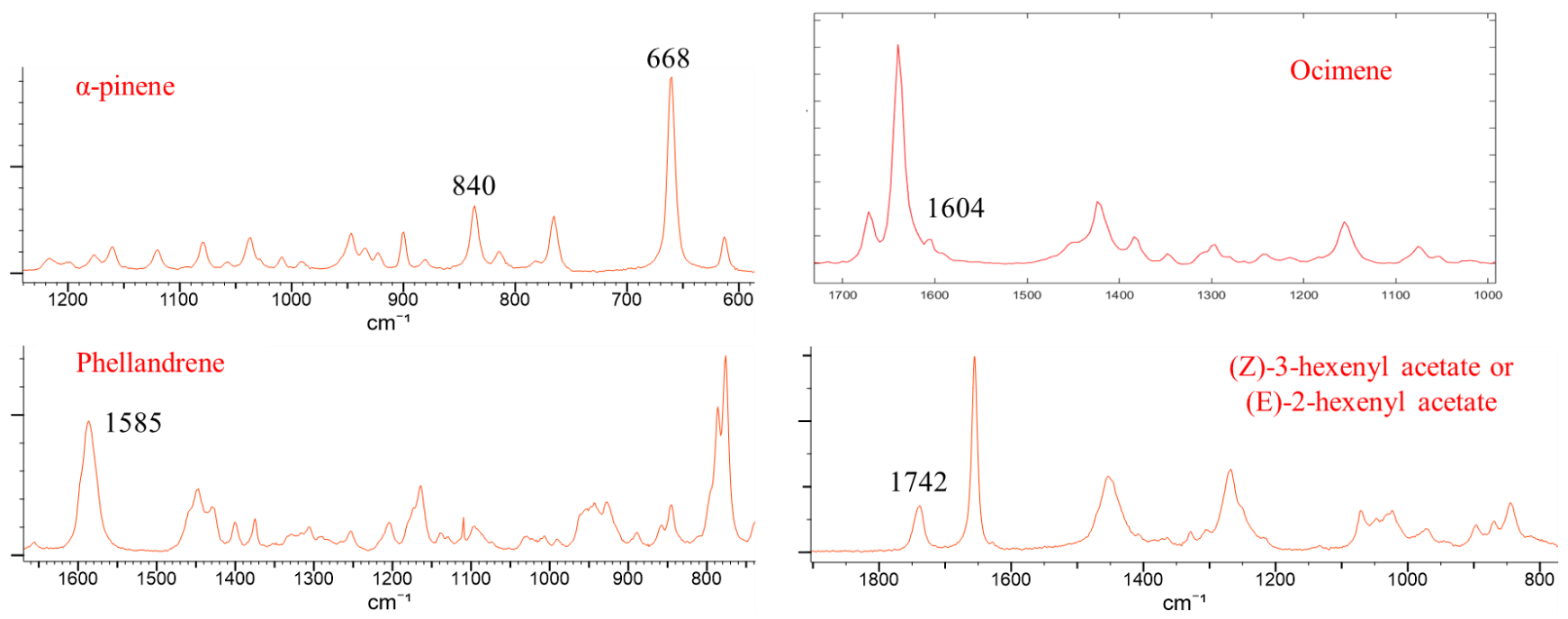

Figure S 3. Raman spectra for standards relevant to Cotton VOC 